\title{
BEHAVIOR ANALYSIS OF TRAFFIC ACCIDENTS WITH HIGH FIDELITY DRIVING SIMULATOR
}

\author{
YANG Fan ${ }^{12}$, ZHANG Kan ${ }^{1 *}$, SUN Xianghong ${ }^{1}$
}

1. State Key Laboratory of Brain and Cognitive Science, Institute of Psychology, Chinese Academy of Sciences, Beijing 100101, China; PH +8610-64851104; Email: yangf@psych.ac.cn

2. Graduate University of Chinese Academy of Sciences, Beijing 100039, China

\begin{abstract}
Unskilled Driving is one of the reasons for traffic accidents. The accidents could be reduced if drivers' skills are tested accurately. In this research, XuanAi QJ4B high fidelity and immersive driving simulator was used as the experimental platform that included testing roads and controlling software. 10 roads based on real roads in China were prepared to be the test ones that including five types of sections: highway, city, country, Mountain and their mixed one. For each road, there were two driving conditions: driving without traffic, driving with heavy traffic and emergencies that probably caused accidents. The software could record the situation of the vehicle and driver such as the time of finishing task, times of out of road, times of collision and so on. 19 drivers participated in the test, and then evaluated driving skills by each other. Comparing the driving situation with or without traffic and analyzing the roads we found out that the time of finishing task and times of out of road were sensitive to distinguish the drivers. In addition, under the situation with heavy traffic, comparing the behavior of accident drivers and behavior of drivers without accidents, the result showed that city road, country road and mountain road were sensitive to distinguish the drivers.
\end{abstract}

Key words: traffic safety, driving Simulator, driving experience, driving behavior

\section{Introduction}

Traffic accidents are more and more in nowadays, the reasons are variable, but about $74 \%$ of them can be attributed to driver failures in China, the percent is greater than accidents of roads (19\%), and accidents of vehicle (7\%) (Chinese ministry of public security, 2007). The further reasons involved fatigue, age, gender, driving skills and experience, and so on. If we can test drivers' driving skills accurately and train the unskilled drivers, many traffic accidents may not happen.

Driving experience is one of the factors influence driving skills, many researches showed that novice drivers have more accidents than experienced ones, because the former have less driving experience (Joel Monárrez-Espino, Marie Hasselberg and Lucie Laflamme, 2005), and eye movement research informed that novice drivers could not scan multiple locations in the visual scene for sources of potential danger (Peter Chapman, Geoffrey Underwood and Katharine Roberts, 2002).

Besides driving experience, the author tried to find other factors, and built a system to test driving skills. The driving simulator was used to build this system.

\footnotetext{
* Corresponding author. Institute of Psychology, Chinese Academy of Sciences, Beijing 100101, China. Email: zhangk@psych.ac.cn
} 
Compared to real vehicle, the merit of using simulator was obvious; it saved time and costs, no real traffic accidents, no limit of weather, field and oil plants. Driving simulator can be categorized to three groups, research, amusement and training (Sunheart, 2006). The researched ones are high fidelity, immersive, have full function and a large field of view. Researches suggested that, if driving simulators have a large field of view, longitudinal speed could be estimated correctly from visual information (Andras Kemeny \& Francesco Panerai, 2003)

The aim of this study was to build a system based on high fidelity and immersive driving simulator to test driving skills accurately.

\section{Method}

\subsection{Apparatus}

XuanAi QJ4B was a high fidelity and immersive driving simulator(see figure 1), which contained a truck's head (the full truck is $12 \mathrm{~m}$ in length), three screens that made the field of view be about 180 degree, six servo-arms that got it more flexible, and controlling PCs.

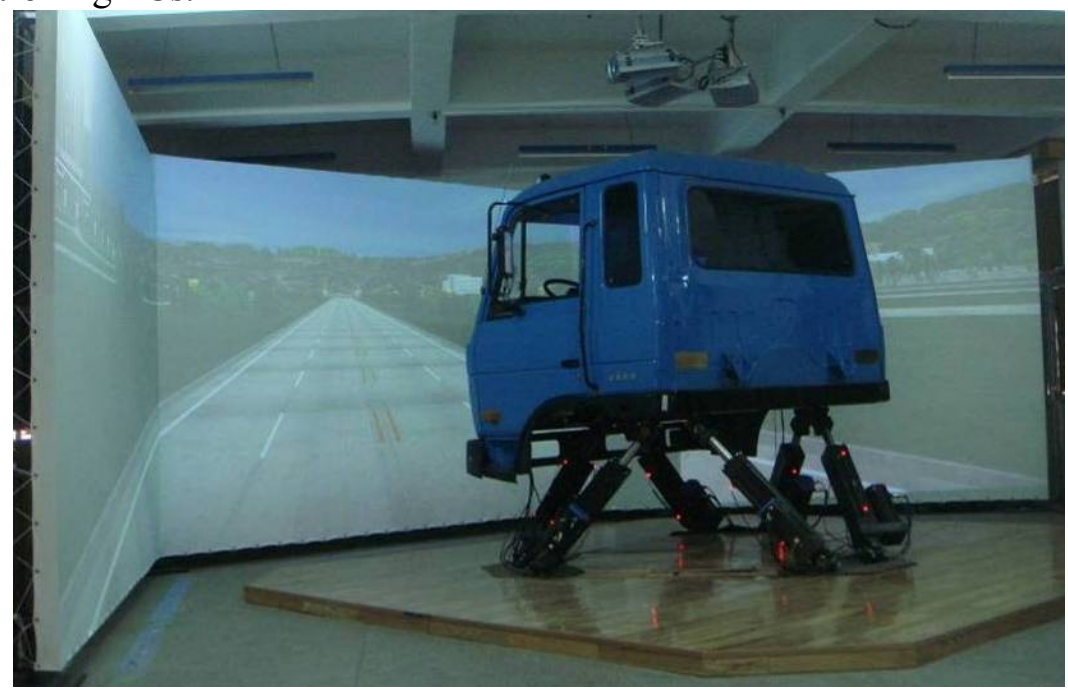

Figure 1 XuanAi QJ4B driving simulator

\section{2 roads and controlling software}

Based on real roads in China, ten roads were created, which included five types of section: highway, city, country, Mountain and their mixed one (see figure 2, 3, 4, 5, 6). Six roads had only one type; the other had two types mixed.

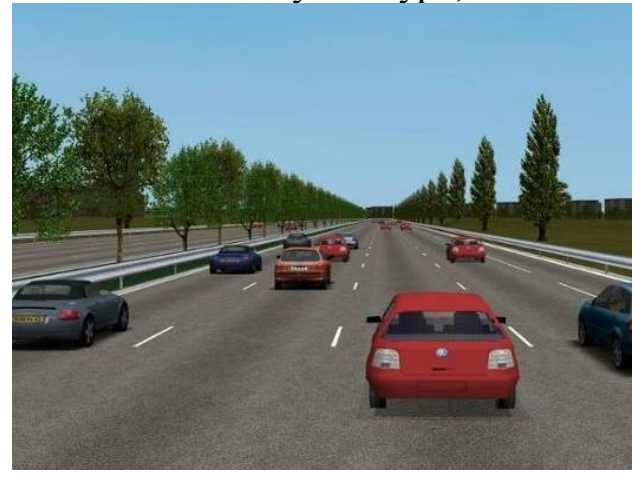

Figure 2 highway

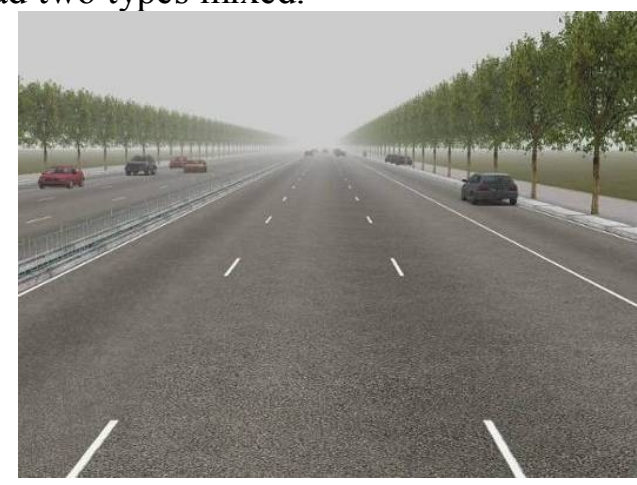

Figure 3 city roads 


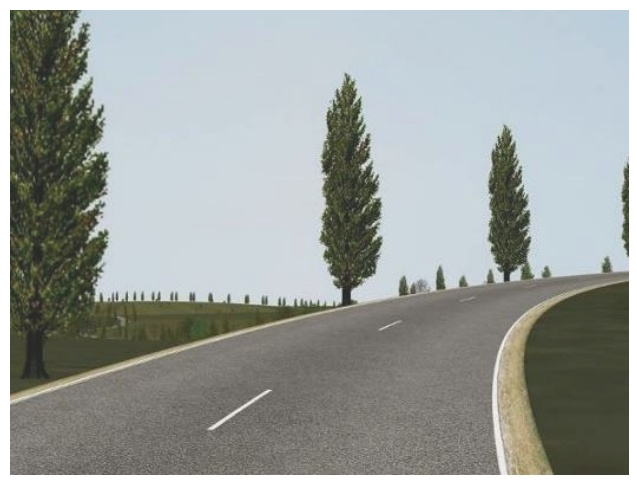

Figure 4 mountain roads

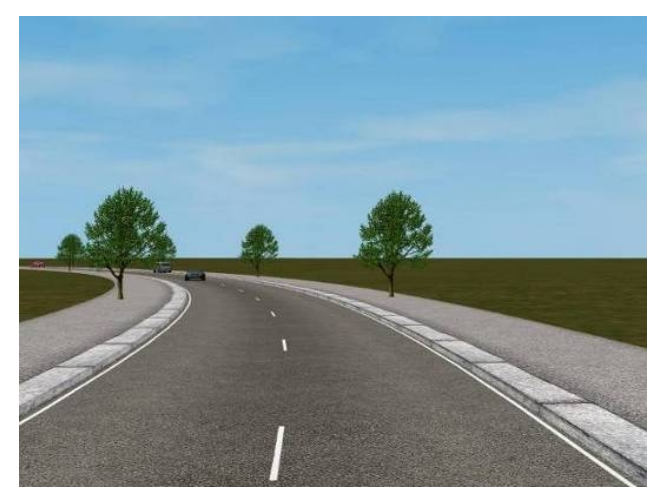

Figure 5

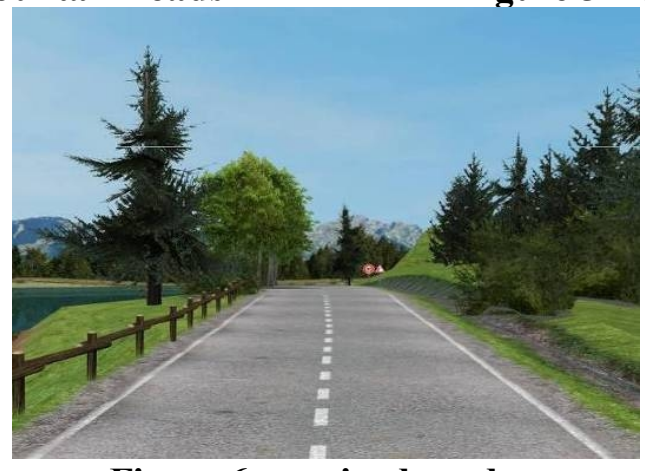

Figure 6 mixed roads

For each road, there were two driving conditions: driving without traffic; and driving with heavy traffic and 2 to 5 emergencies that probably caused accidents. The emergencies included that pedestrians walked across the road, a car blocked dead ahead, collision in the intersection, traffic jam, and so on. So there were 20 driving routes for testing in all.

There were enough traffic signs inducting drivers, such as turn left, turn right, test started, and test over, and so on. If drivers drove out of roads, there would be a sign showed in the center of screen. And if drivers crashed, the sound, pictures and the servo-device would give drivers immersive environment.

The controlling software could control the simulator and record the situation of the vehicle and driver every $50 \mathrm{~ms}$, such as the time of finishing task, times of out of road, times of collision, speed, acceleration, the angle of steering wheel, vehicle RPM, state of horn, and so on.

\section{3 participants}

19 driving soldiers participated in the experiment (they were all male, the simulator was in barrack, strangers were not admitted), their ages ranged from 18 to 35 years, their driving experience were divided into four groups, less than one year, one to three years, three to five years, and above five years.

\section{3 procedure}

Each driver completed all driving routes (including 20 routes, about 3 minutes each), and rested about 5 minutes every 4 routes. The participants were instructed to finish task as soon as possible in condition of being safe, and they should keep in the center of lane where they were driving.

After driving test, the participants ranked their driving skills each other. 


\section{Results}

\section{1 ranks}

Summed up the ranking grades of each driver, and then divided drivers into three parts by their grades, good skills ( 6 persons), middle skills (6 persons), and bad skills (7 persons).

\section{2 index of driving skills}

Driving skills contained many factors, five variables were chosen to analyze in this study, time of finishing each road (referred as time), times of out of each road (referred as out), times of collision (referred as collision), times of deviating the center of lane(referred as away) and times of ignition(referred as fire).

The variables were normalized (see figure 7), and then analyzed by applying one-way ANOVA. The time of finishing each road was remarkably different in three ranks $(F=5.674, p=0.014)$; the times of out of each road was a little different in three ranks $(F=2.763, p=0.093)$, the other three variables were not different in three ranks.

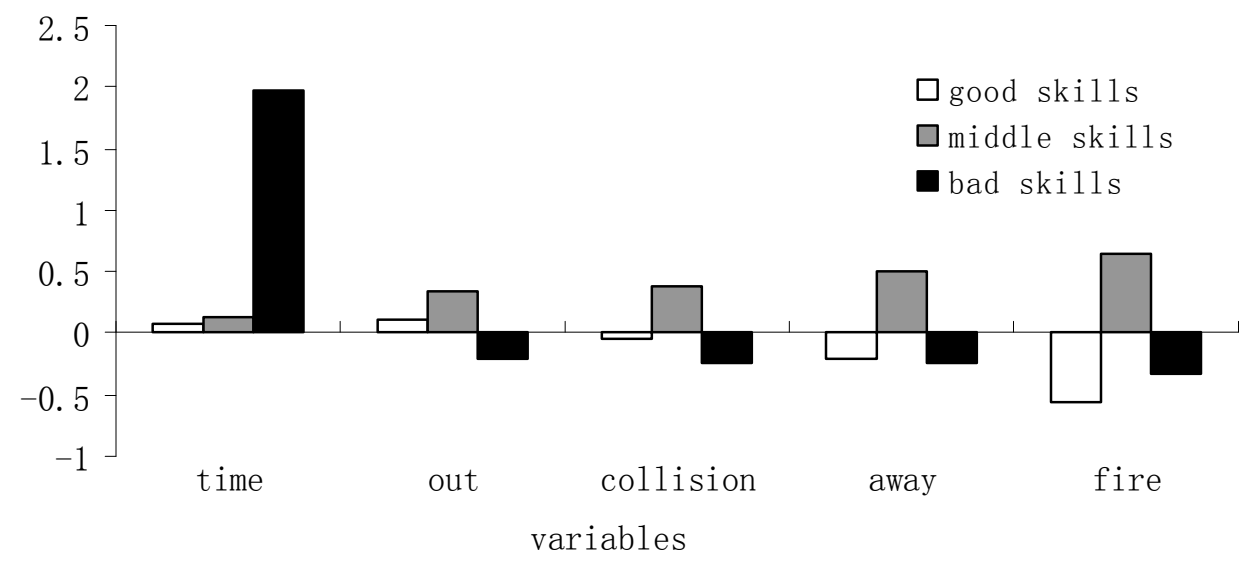

Figure 7 normalized variables in three ranks

Driving experience (referred as years) was also analyzed by Kruskal-Wallis Test, it was remarkably different in three ranks (chi-square $(2)=9.656, p=0.008$ ).

\section{2 roads}

Not every road was good to test drivers' driving skills; we also needed to find out which road was appropriate. Since we had find out time, out and years could differentiate drivers, so we use these variables to select roads.

Some roads were remarkably different in time, city roads with traffic $(F=5.058$, $p=0.02)$, mountain road with traffic $(F=4.653, p=0.026)$, mountain and city mixed road without traffic $(F=5.491, p=0.015)$; Some roads were a little different in time, country road with traffic $(F=2.941, p=0.082)$, mountain and city mixed road with traffic $(F=3.34, p=0.061)$.

Only one road was different in out, mountain and country mixed road without traffic $(F=3.012, p=0.078)$.

In sum, seven roads were effective.

\section{Discussions}

Time of finishing each road and driving experience could differentiate drivers remarkably, the reason was that drivers with better driving skills often had more 
driving experience, and always could control vehicle better, so they finished the same task in less time.

Times of out of road could inform drivers' stability of driving, drivers with better skills also drove more steadily, had less times of out of road.

Maybe very hard roads could differentiate drivers better, city road with traffic had more vehicle, buildings, people, and traffic jam and so on, and mountain road with traffic was very rough, tortuous and rugged, it was hard to drive.

However, there were still some problems, building a driving skills tested system need more participants, and then the system would be more accurate.

\section{Conclusions}

This study had built a system with seven effective roads and three variables to test drivers' driving skills. It could help driving school or driving licenses issued organization evaluate drivers, and help drives' skills improve to reduce traffic accidents.

\section{References}

Andras Kemeny \& Francesco Panerai. (2003)."Evaluating perception in driving simulation experiments". Trends in Cognitive Sciences, Volume 7, Issue 1, January 2003, Pages 31-37

Beijing sunheart simulator company. (2006)."The categories of driving simulator". http://www.bjxa.com/theory/sorts/sorts01.jsp

Hinese ministry of public security. (2007). "Section 5: responsible ones." A summary of traffic accidents on roads in China. Beijing, 5.1-5.15.

Joel Monárrez-Espino, Marie Hasselberg and Lucie Laflamme. (2006). "First year as a licensed car driver: Gender differences in crash experience". Safety Science,44, $75-85$.

Peter Chapman, Geoffrey Underwood and Katharine Roberts. (2002)."Visual search patterns in trained and untrained novice drivers". Transportation Research Part F: Traffic Psychology and Behaviour, 5(2), 157-167. 